PROCEEDINGS OF THE

AMERICAN MATHEMATICAL SOCIETY

Volume 137, Number 10, October 2009, Pages 3523-3531

S 0002-9939(09)09907-9

Article electronically published on June 29, 2009

\title{
CONSTRUCTING CONVEX PLANES IN THE PANTS COMPLEX
}

\author{
JAVIER ARAMAYONA, HUGO PARLIER, AND KENNETH J. SHACKLETON \\ (Communicated by Alexander N. Dranishnikov)
}

\begin{abstract}
Our main theorem identifies a class of totally geodesic subgraphs of the 1-skeleton of the pants complex, referred to as the pants graph, each isomorphic to the product of two Farey graphs. We deduce the existence of many convex planes in the pants graph of any surface of complexity at least 3 .
\end{abstract}

\section{INTRODUCTION}

Let $\Sigma$ be a compact, connected and orientable surface, possibly with non-empty boundary, of genus $g(\Sigma)$ and $|\partial \Sigma|$ boundary components. After Hatcher-Thurston [7, to the surface $\Sigma$ one may associate a simplicial graph $\mathcal{P}(\Sigma)$, the pants graph, whose vertices are all the pants decompositions of $\Sigma$ and any two vertices are connected by an edge if and only if they differ by an elementary move; see Section 2.2 for an expanded definition. In other words, the pants graph is precisely the 1-skeleton of the 2-dimensional cell complex commonly referred to in the literature as the pants complex. The pants graph is connected [7], and one may introduce the canonical path-metric $d$ on $\mathcal{P}$ by first assigning length 1 to each edge and then regarding the result as a geodesic metric space.

The pants graph, with its own geometry, is a fundamental object to study. Brock [3] revealed deep connections with hyperbolic 3-manifolds and proved the pants graph is the correct combinatorial model for the Weil-Petersson metric on Teichmüller space, for the two are quasi-isometric. The isometry group of $(\mathcal{P}, d)$ is also correct in so far as the study of surface groups is concerned, for Margalit [8] proved it is almost always isomorphic to the mapping class group of $\Sigma$. In addition, Masur-Schleimer 11 proved the pants graph of any closed surface of genus at least 3 to be one-ended. With only finitely many exceptions, the pants graph is not hyperbolic in the sense of Gromov [4] or even strongly relatively hyperbolic [2, 5] in any of the standard senses. A lack of negative curvature on a large-scale in even a relative sense is often an impediment to studying both the synthetic and coarse geometry of a metric space.

In [1], the authors prove that every subgraph of $\mathcal{P}$ isomorphic to the Farey graph is in fact totally geodesic in $(\mathcal{P}, d)$. The purpose of this paper is to study the extrinsic geometry of another class of subgraphs of the pants graph, each determined

Received by the editors February 27, 2007, and, in revised form, October 26, 2008.

2000 Mathematics Subject Classification. Primary 57M50; Secondary 05C12.

Key words and phrases. Pants complex, Weil-Petersson metric.

The first author was partially supported by a short-term research fellowship at the Université de Provence, and the third author by a long-term JSPS postdoctoral fellowship, number P06034.

(C)2009 American Mathematical Society Reverts to public domain 28 years from publication 
by 2-handle multicurves as defined at the end of Section 2.1 and as such isomorphic to the product of two Farey graphs. This is a special case of Conjecture 5 from [1].

Theorem 1. Let $\Sigma$ be a compact, connected and orientable surface, and denote by $Q$ any 2 -handle multicurve on $\Sigma$. Then, $\mathcal{P}_{Q}$ is totally geodesic in $\mathcal{P}(\Sigma)$.

The Weil-Petersson metric on Teichmüller space is not complete, since the length of a simple closed geodesic can converge to zero on bounded subsets of Teichmüller space [13, 6]. Indeed, Masur [9] showed that the completion of the Weil-Petersson metric is homeomorphic to the augmented Teichmüller space, obtained by suitably extending every Fenchel-Nielsen co-ordinate system to admit zero lengths. The completion thus carries the structure of a stratified space, where by definition each stratum assigns zero length to its corresponding multicurve. These strata are all convex 12; in particular those strata corresponding to 2-handle multicurves are all convex. For any given 2-handle multicurve $Q$, the quasi-isometry offered in Theorem 1.1 of Brock [3] may be chosen so as to map the stratum corresponding to $Q$ onto the subgraph $\mathcal{P}_{Q}$ of the pants graph. It follows that such subgraphs are uniformly quasi-isometrically embedded in the pants graph. This fact is also implicit in the earlier work of Masur-Minsky [10].

Theorem 1 thus offers a complete analogy between the geometry of the 2-handle subgraphs in the pants graph and the geometry of the corresponding strata lying in the completed Weil-Petersson space. In order to prove Theorem 1 we shall project paths in the pants graph to paths in the given 2-handle subgraph of no greater length. All the notation of Theorem 2 will be explained in Section 2, but for now we point out the finite set of pants decompositions $\pi_{Q}(\nu)$ is reminiscent of the subsurface projection after Masur-Minsky 10 of a pants decomposition $\nu$ to the subgraph $\mathcal{P}_{Q}$ determined by the 2 -handle multicurve $Q$. The intrinsic metric on the graph $\mathcal{P}_{Q}$, assigning length 1 to each edge, is denoted by $d_{Q}$.

Theorem 2. Let $\Sigma$ be a compact, connected and orientable surface and denote by $Q$ any 2-handle multicurve on $\Sigma$. Let $\left(\nu_{0}, \ldots, \nu_{n}\right)$ be a path in the pants graph $\mathcal{P}(\Sigma)$. For each index $i \leq n-2$ and for each $\omega_{i} \in \pi_{Q}\left(\nu_{i}\right)$, there exists an integer $j \in\{1,2\}$ and a pants decomposition $\omega_{i+j} \in \pi_{Q}\left(\nu_{i+j}\right)$ of $\Sigma$ such that $d_{Q}\left(\omega_{i}, \omega_{i+j}\right) \leq j$.

To the authors' knowledge, it has yet to be decided whether there exists a distance non-increasing projection from the whole pants graph to any one of its subgraphs corresponding to a 2-handle multicurve. In the absence of an affirmative result, Theorem 2 may well hold independent interest.

Let us indicate a few consequences of Theorem 1. First, by considering a pair of bi-infinite geodesic paths, one in either factor Farey graph for a 2-handle multicurve, we deduce the following. By a plane we shall mean a graph isomorphic to the Cayley graph of the group $\mathbb{Z} \oplus \mathbb{Z}$ with standard generating set.

Corollary 3. Let $\Sigma$ be a compact, connected and orientable surface of complexity at least 3 . Then, $\mathcal{P}(\Sigma)$ contains infinitely many convex planes, each contained in a 2-handle subgraph.

These planes are not all totally geodesic, since there exist bi-infinite geodesic paths in a Farey graph which are not totally geodesic. As we note in Lemma 9 towards the end of Section 5, in the pants graph of the 6-holed sphere, the 3-holed torus, and the closed surface of genus 2 there are no other convex planes: the 
vertices of any given convex plane all contain a common separating curve whose complement in $\Sigma$ is the union of two surfaces neither of which is a 3-holed sphere.

The plan of this paper is as follows. In Section 2 we recall all the terminology we need, much of which is already standard. In Section 3 we give an elementary proof to Theorem 2. In Section 4 we apply Theorem 2 to give an elementary proof to Theorem 1. In Section 5 we prove Corollary 3 and classify the convex planes in the pants graph of any one of the complexity 3 surfaces.

\section{BACKGROUND AND DEFINITIONS}

We supply all the background and terminology needed both to understand the statements of our main results, and to make sense of their proofs. Throughout, we define a loop on $\Sigma$ as the homeomorphic image of a standard circle.

2.1. Curves and multicurves. A loop on $\Sigma$ is said to be trivial if it bounds a disc and peripheral if it bounds an annulus whose other boundary component belongs to $\partial \Sigma$. For a non-trivial and non-peripheral loop $c$, we shall denote by $[c]$ its free homotopy class. A curve is by definition the free homotopy class of a non-trivial and non-peripheral loop. Given any two curves $\alpha$ and $\beta$, their intersection number $\iota(\alpha, \beta)$ is defined equal to $\min \{|\mathrm{a} \cap \mathrm{b}|: \mathrm{a} \in \alpha, \mathrm{b} \in \beta\}$.

We shall say two curves are disjoint if they have zero intersection number, and otherwise say they intersect essentially. A pair of curves $\{\alpha, \beta\}$ is said to fill the surface $\Sigma$ if $\iota(\delta, \alpha)+\iota(\delta, \beta)>0$ for every curve $\delta$. In other words, every curve on $\Sigma$ intersects at least one of $\alpha$ and $\beta$ essentially.

A multicurve is a (possibly empty) collection of distinct and disjoint curves, and the intersection number for a pair of multicurves is to be defined additively. While in the literature the definition of a multicurve does at times permit parallel copies of any given curve, this is not the case here. We denote by $\kappa(\Sigma)$ the cardinality of any maximal multicurve on $\Sigma$, equal to $3 g(\Sigma)+|\partial \Sigma|-3$, and refer to this as the complexity of $\Sigma$. Note, only two surfaces are of complexity 1 , namely the 4-holed sphere and the 1-holed torus.

Given a set of disjoint loops $L$, such as the boundary of some subsurface of $\Sigma$, we denote by $[L]$ the multicurve maximal among all multicurves whose every curve is represented by some element of $L$. We reserve ourselves the freedom to at times regard a curve or a multicurve as a class, or a set of classes, or as the disjoint union of simple closed loops realised on $\Sigma$ in general position as need dictates and without making further comment.

We shall say a multicurve $\omega$ has rank $k$, for some non-negative integer $k$, if $|\omega|=\kappa(\Sigma)-k$. We shall say a rank 2 multicurve $Q$ is a 2-handle multicurve if the complement of every simple representative of $Q$ contains two complexity 1 components, each with every boundary component trivial in the relative integral homology group $H_{1}(\Sigma, \partial \Sigma)$.

2.2. Pants decompositions. A pants decomposition of a surface is a maximal collection of distinct and disjoint curves, in other words a maximal multicurve. Two pants decompositions $\mu$ and $\nu$ are said to be related by an elementary move if $\mu \cap \nu$ is a rank 1 multicurve and the remaining two curves together either fill a 4-holed sphere and intersect twice or fill a 1-holed torus and intersect once; consider Figure 1, 


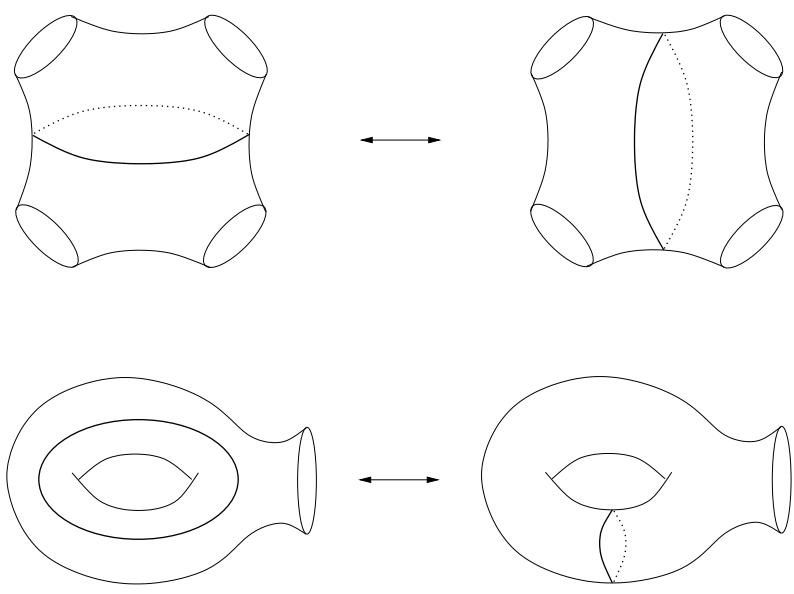

Figure 1. The two types of elementary move.

2.3. Arcs. An arc on $\Sigma$ is the homotopy class, modulo $\partial \Sigma$, of an embedded interval ending on $\partial \Sigma$ that does not bound a disc with $\partial \Sigma$. There are broadly two types of arc: those that end on only one component of $\partial \Sigma$, referred to as waves, and those that end on two different components of $\partial \Sigma$, referred to as seams. Owing to features particular to 2-handle multicurves we need only consider waves.

We may similarly speak of two arcs, or an arc and a curve, intersecting or being disjoint.

2.4. Graphs and paths. For us, a path in a graph shall be a finite sequence of vertices such that any consecutive pair spans an edge; one can recover a topological path by joining up the dots. A geodesic is then a path realising distance. A subgraph $F$ of a graph $G$ is said to be totally geodesic if every geodesic in $G$ whose two endpoints belong to $F$ is entirely contained in $F$. A subgraph $F$ of a graph $G$ is said to be convex if any two vertices of $F$ are connected by a geodesic entirely contained in $F$. Any totally geodesic subgraph is convex, though a convex subgraph need not be totally geodesic. Finally, the product of two graphs $G$ and $H$, denoted $G * H$, is the graph with vertex set $V(G) \times V(H)$ and two distinct vertices $\left(x_{1}, x_{2}\right)$ and $\left(y_{1}, y_{2}\right)$ are connected by an edge if and only if either $x_{1}$ and $y_{1}$ span an edge in $G$ and $x_{2}=y_{2}$, or $x_{2}$ and $y_{2}$ span an edge in $H$ and $x_{1}=y_{1}$. The double of the graph $G$ is defined to be the graph $G * G$.

2.5. Subsurface projections. A subsurface $Y$ of $\Sigma$ is said to be essential if no component of $Y$ is parallel to a component of $\partial \Sigma$ and each component of $\partial Y$ either determines a curve or is parallel to a component of $\partial \Sigma$. If every loop representing a curve $\alpha$ has non-empty intersection with an essential subsurface $Y$ we say $\alpha$ and $Y$ intersect; otherwise we say they are disjoint. We may similarly speak of a multicurve intersecting or being disjoint from $Y$.

Let $Y$ denote any complexity 1 essential subsurface of $\Sigma$. For $\alpha$ any curve intersecting $Y$ denote by $b$ a component of the intersection $\alpha \cap Y$. Regardless of whether $b$ is an arc or a curve, there exists only one curve on $Y$ disjoint from $b$ and such a curve shall be referred to as a projection of $\alpha$. The intersection $b$ need not 
be unique, up to homotopy, but the set of all such projections, each counted once, is finite and depends only on $\alpha$ and the isotopy class of the surface $Y$. We denote this set by $\pi_{Y}(\alpha)$. If $\alpha$ is a curve disjoint from $Y$, we define $\pi_{Y}(\alpha)$ to be equal to the empty set. For a multicurve $\nu$ we may similarly define $\pi_{Y}(\nu)$, a finite set of curves. The set $\pi_{Y}(\nu)$ is an instance of the subsurface projection as defined by Masur-Minsky in Section 1.1 of [10].

Let $Q$ be a 2-handle multicurve and denote by $Y_{1}$ and $Y_{2}$ the two non-isotopic essential complexity 1 subsurfaces of $\Sigma$ disjoint from $Q$. For any multicurve $\nu$ on $\Sigma$ intersecting both $Y_{1}$ and $Y_{2}$ we define $\pi_{Q}(\nu)$ to be equal to the finite set of pants decompositions $\left\{\left\{\delta_{1}, \delta_{2}\right\} \cup Q: \delta_{1} \in \pi_{Y_{1}}(\nu), \delta_{2} \in \pi_{Y_{2}}(\nu)\right\}$. For any multicurve $\nu$ on $\Sigma$ intersecting only $Y_{1}$, we define $\pi_{Q}(\nu)$ to be equal to the finite set of multicurves $\left\{\left\{\delta_{1}\right\} \cup Q: \delta_{1} \in \pi_{Y_{1}}(\nu)\right\}$. For any multicurve $\nu$ on $\Sigma$ disjoint from both $Y_{1}$ and $Y_{2}$ we define $\pi_{Q}(\nu)$ equal to $\{Q\}$.

We note that if $\nu$ is a multicurve intersecting both $Y_{1}$ and $Y_{2}$, as every pants decomposition of $\Sigma$ must, then each element of $\pi_{Q}(\nu)$ is a pants decomposition containing $Q$ and, as such, a vertex of $\mathcal{P}_{Q}$. Conversely, if $\pi_{Q}(\nu)$ contains at least one pants decomposition, then $\nu$ intersects both $Y_{1}$ and $Y_{2}$ and every element of $\pi_{Q}(\nu)$ is a pants decomposition. In other words, for the most part the reader may safely restrict $\pi_{Q}$ to a map from the set of all multicurves intersecting both $Y_{1}$ and $Y_{2}$ to the power set of the set of all pants decompositions of $\Sigma$. This map further restricts to an injection on the subgraph $\mathcal{P}_{Q}$.

Remark. If $\delta \subset Y_{1}$ is a curve and $\nu$ is a pants decomposition disjoint from $\delta$, then $\delta$ is contained in $\nu$ and thus in the unique pants decomposition belonging to $\pi_{Q}(\nu)$. In particular, if $\nu \in \mathcal{P}_{Q}$ and $\nu^{\prime} \notin \mathcal{P}_{Q}$ are adjacent, then $\pi_{Q}\left(\nu^{\prime}\right)=\nu$. This "orthogonality" property of projections will be crucial to the proof of Theorem 1 offered in Section 4.

\section{Proof of Theorem 2}

We proceed by constructing inductively a projection for the given path to $\mathcal{P}_{Q}$, starting from the initial pants decomposition $\nu_{0}$. As the projection sets of each $\nu_{i}$ typically comprise more than one pants decomposition, at times pairwise distance 2 apart, this will involve making a careful choice of image at each step while ensuring that the path constructed in $\mathcal{P}_{Q}$ is of no greater length than the original path.

The three statements given below together adequately describe the ways in which the projection sets of two or three consecutive pants decompositions can align themselves relative to one another.

Lemma 4. Let $Q$ be a rank 1 multicurve on $\Sigma$, and denote by $Y$ the essential complexity 1 subsurface of $\Sigma$ disjoint from $Q$. For two disjoint waves or curves $a_{1}$ and $a_{2}$ on $Y$, denote by $\alpha_{1}$ and $\alpha_{2}$ the unique curves on $Y$ such that $\iota\left(\alpha_{1}, a_{1}\right)=0$ and such that $\iota\left(\alpha_{2}, a_{2}\right)=0$. Then, $d\left(\left\{\alpha_{1}\right\} \cup Q,\left\{\alpha_{2}\right\} \cup Q\right) \leq 1$.

Proof. If at least one of $a_{1}$ and $a_{2}$ is a curve, then $\alpha_{1}$ and $\alpha_{2}$ are equal and $d\left(\left\{\alpha_{1}\right\} \cup\right.$ $\left.Q,\left\{\alpha_{2}\right\} \cup Q\right)=0$. Otherwise, $\alpha_{1}$ and $\alpha_{2}$ are either equal or intersect minimally, and as such $d\left(\left\{\alpha_{1}\right\} \cup Q,\left\{\alpha_{2}\right\} \cup Q\right) \leq 1$.

In what follows we make repeated use of the above observation that a pair of disjoint waves projects to a pair of curves either equal or intersecting minimally. 
Lemma 5. Let $Q$ be a 2-handle multicurve on $\Sigma$, and denote by $Y_{1}$ and $Y_{2}$ the two non-isotopic complexity 1 subsurfaces of $\Sigma$ disjoint from $Q$. Let $\nu_{0}$ and $\nu_{1}$ be two vertices of $\mathcal{P}(\Sigma)$ such that $d\left(\nu_{0}, \nu_{1}\right)=1$. For $\omega_{0} \in \pi_{Q}\left(\nu_{0}\right)$, if there exists $\omega_{1} \in \pi_{Q}\left(\nu_{1}\right)$ such that $\omega_{0} \cap \omega_{1}$ properly contains $Q$, then there exists $\omega_{1}^{\prime} \in \pi_{Q}\left(\nu_{1}\right)$ such that $d\left(\omega_{0}, \omega_{1}^{\prime}\right) \leq 1$.

Proof. Let $\omega_{0} \in \pi_{Q}\left(\nu_{0}\right)$, and suppose there exists $\omega_{1} \in \pi_{Q}\left(\nu_{1}\right)$ such that $\omega_{0} \cap \omega_{1}$ properly contains $Q$. If $d\left(\omega_{0}, \omega_{1}\right) \geq 2$, then there exists a curve in $\nu_{0}$ intersecting both $Y_{1}$ and $Y_{2}$ and projecting to $\omega_{0}$. The rank 1 multicurve $\nu_{0} \cap \nu_{1}$ therefore intersects both $Y_{1}$ and $Y_{2}$, and so there exists a pants decomposition $\omega_{1}^{\prime} \in$ $\pi_{Q}\left(\nu_{0} \cap \nu_{1}\right)$. As such, $\omega_{1}^{\prime} \in \pi_{Q}\left(\nu_{0}\right)$ and so $d\left(\omega_{0}, \omega_{1}^{\prime}\right) \leq 1$. If on the other hand $d\left(\omega_{0}, \omega_{1}\right) \leq 1$, then we may define $\omega_{1}^{\prime}$ to be equal to $\omega_{1}$.

Lemma 6. Let $Q$ be a 2-handle multicurve on $\Sigma$, and denote by $Y_{1}$ and $Y_{2}$ the two non-isotopic essential complexity 1 subsurfaces of $\Sigma$ disjoint from $Q$. Let $\left(\nu_{0}, \nu_{1}, \nu_{2}\right)$ be any geodesic in $\mathcal{P}(\Sigma)$ of length 2 , and let $\omega_{0} \in \pi_{Q}\left(\nu_{0}\right)$. If $\omega_{0} \cap \omega_{1}$ is equal to $Q$ for each $\omega_{1} \in \pi_{Q}\left(\nu_{1}\right)$, then the multicurve $\nu_{0} \cap \nu_{1} \cap \nu_{2}$ intersects both $Y_{1}$ and $Y_{2}$.

Proof. As $\omega_{0} \cap \omega_{1}$ is equal to $Q$ for each $\omega_{1} \in \pi_{Q}\left(\nu_{1}\right)$, there exists a unique curve $\delta \in \nu_{0}$ intersecting both $Y_{1}$ and $Y_{2}$ such that $\omega_{0} \in \pi_{Q}(\delta)$. (See Figure 2.) There also exists a curve $\delta^{\prime}$ disjoint from $Y_{1}$ but intersecting $Y_{2}$ such that any multicurve disjoint from both $\left[\partial Y_{1}\right]$ and $\delta$ is also disjoint from $\delta^{\prime}$, and such that $\omega_{0} \cap \omega$ properly contains $Q$ for some (rank 1 multicurve) $\omega \in \pi_{Q}\left(\delta^{\prime}\right)$. Indeed, one may explicitly construct such a curve by suitably projecting $\delta$ to the complement of $Y_{1}$ on $\Sigma$.

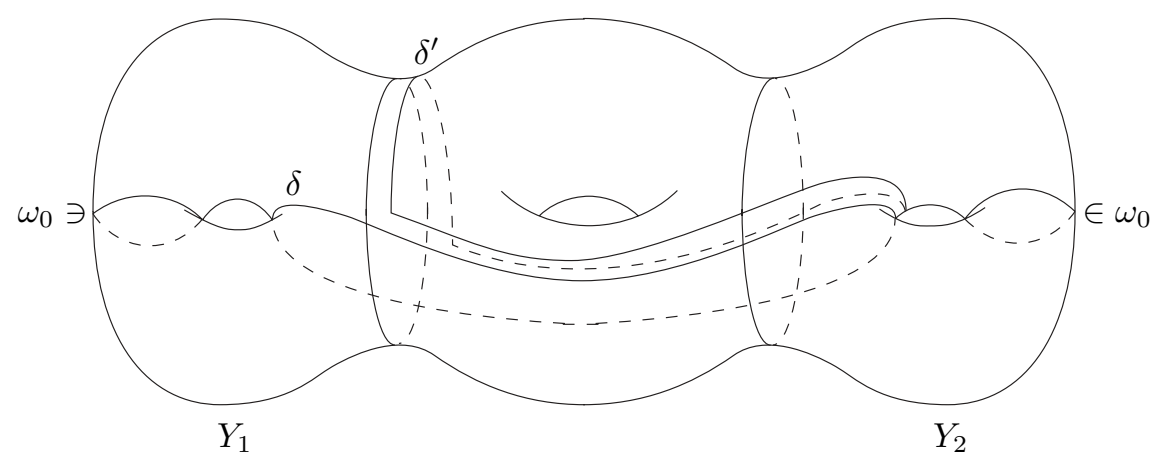

Figure 2. The curve $\delta$ projects to $\omega_{0}$ and $\delta^{\prime}$.

Let $R$ denote the multicurve $\nu_{0} \cap \nu_{1} \cap \nu_{2}$, noting $|R| \geq \kappa(\Sigma)-2$, and suppose for contradiction that $R$ does not intersect $Y_{1}$. We now note that $\delta^{\prime}$ is then distinct and disjoint from every curve in $R$, for respectively the multicurve $\omega_{0} \cap \omega_{1}$ is only equal to $Q$ for each $\omega_{1} \in \pi_{Q}\left(\nu_{1}\right)$, and hence for each $\omega_{1} \in \pi_{Q}(R)$, and $R$ is disjoint from both $\left[\partial Y_{1}\right]$ and $\delta$. It follows that $R \sqcup\left\{\delta^{\prime}\right\}$ is a multicurve disjoint from $Y_{1}$ and, since it cannot contain $\left[\partial Y_{1}\right]$, as such has cardinality at most $\kappa(\Sigma)-2$. Thus,

$$
|R|=\left|\left(R \sqcup\left\{\delta^{\prime}\right\}\right)-\left\{\delta^{\prime}\right\}\right|=\left|R \sqcup\left\{\delta^{\prime}\right\}\right|-\left|\left\{\delta^{\prime}\right\}\right| \leq \kappa(\Sigma)-2-1=\kappa(\Sigma)-3 .
$$

To be more succinct, $|R| \leq \kappa(\Sigma)-3$. We therefore have two incompatible estimates for the cardinality of $R$, and this is a contradiction. 
A parallel argument applies to $Y_{2}$, and the statement of the lemma therefore holds.

We denote by $Y_{1}$ and $Y_{2}$ the two essential non-isotopic complexity 1 subsurfaces of $\Sigma$ disjoint from $Q$. Let $\omega_{0}$ denote any element of $\pi_{Q}\left(\nu_{0}\right)$.

Suppose inductively we have chosen the vertex $\omega_{k} \in \pi_{Q}\left(\nu_{k}\right)$, for some $k \geq 0$. If there exists a pants decomposition $\omega \in \pi_{Q}\left(\nu_{k+1}\right)$ such that $\omega_{k} \cap \omega$ is not equal to $Q$, then by Lemma 5 there exists a pants decomposition $\omega^{\prime} \in \pi_{Q}\left(\nu_{k+1}\right)$ such that $d\left(\omega_{k}, \omega^{\prime}\right) \leq 1$. We define $\omega_{k+1}$ to be equal to $\omega^{\prime}$.

We now consider the sole remaining case, that $\omega_{k} \cap \omega$ is equal to $Q$ for every pants decomposition $\omega \in \pi_{Q}\left(\nu_{k+1}\right)$. By Lemma 6 the multicurve $\nu_{k} \cap \nu_{k+1} \cap \nu_{k+2}$, denoted $R$, intersects both $Y_{1}$ and $Y_{2}$. Taking one intersection of $R$ with $Y_{1}$ and then with $Y_{2}$, we may construct pants decompositions $\omega_{k+1}$ and $\omega_{k+2}$ of $\mathcal{P}_{Q}$ such that $\omega_{k} \cap \omega_{k+1}$ and $\omega_{k+1} \cap \omega_{k+2}$ are both rank 1 multicurves, and where $\omega_{k+2} \in$ $\pi_{Q}(R) \subseteq \pi_{Q}\left(\nu_{k+2}\right)$. Note though, $\omega_{k+1}$ need not be contained in $\pi_{Q}\left(\nu_{k+1}\right)$. As each intersection of $\omega$ with $Y_{1}$ and with $Y_{2}$ is either a wave or a curve, by Lemma 4 we have in turn $d\left(\omega_{k}, \omega_{k+1}\right) \leq 1$ and $d\left(\omega_{k+1}, \omega_{k+2}\right) \leq 1$. Thus $d\left(\omega_{k}, \omega_{k+2}\right) \leq$ $d\left(\omega_{k}, \omega_{k+1}\right)+d\left(\omega_{k+1}, \omega_{k+2}\right) \leq 1+1=2$, and the induction continues from $k+2$. This concludes the proof of Theorem 2 ,

We do not rule out the possibility that the techniques presented above can be adapted to treat all rank 2 multicurves, or to treat "many-handle" multicurves. That said, the number of cases to be considered, arising from the possibility of seams and the number of subsurfaces to project to, is likely to be prohibitive. These more general statements have already been conjectured, being special cases of Conjecture 5 from [1].

\section{Proof of Theorem 1}

Suppose, for contradiction, that $\mathcal{P}_{Q}$ is not totally geodesic for some 2-handle multicurve $Q$. Then, there exists a geodesic $\left(\nu_{0}, \ldots, \nu_{n}\right)$ in $\mathcal{P}(\Sigma)$ such that $\left\{\nu_{0}, \nu_{n}\right\}$ $\subset \mathcal{P}_{Q}$ but $\nu_{j} \notin \mathcal{P}_{Q}$ for each $j \in\{1,2, \ldots, n-1\}$. Applying Theorem 2 inductively, we find an increasing sequence of integers $\left\{k_{1}, k_{2}, \ldots, k_{m}\right\} \subseteq\{1,2, \ldots, n\}$, containing 1 and at least one of $n-1$ and $n$, and a corresponding sequence of pants decompositions $\omega_{k_{j}} \in \pi_{Q}\left(\nu_{k_{j}}\right)$ such that $0<k_{j+1}-k_{j} \leq 2$, for each $j$, and such that $d_{Q}\left(\omega_{k_{j}}, \omega_{k_{j+1}}\right) \leq k_{j+1}-k_{j}$, for each $j$. Necessarily, $\omega_{k_{1}}=\nu_{0}$ and $\omega_{k_{m}}=\nu_{n}$, by the closing remark of Section 2.5. We note that

$$
d_{Q}\left(\omega_{k_{1}}, \omega_{k_{m}}\right) \leq \sum_{j} d_{Q}\left(\omega_{k_{j}}, \omega_{k_{j+1}}\right) \leq \sum_{j} k_{j+1}-k_{j} \leq n-1,
$$

and so it follows that

$$
d\left(\nu_{0}, \nu_{n}\right) \leq d_{Q}\left(\omega_{k_{1}}, \omega_{k_{m}}\right) \leq n-1 .
$$

As $d\left(\nu_{0}, \nu_{n}\right)$ is in fact equal to $n$, this is a contradiction and the statement of Theorem 1 follows.

\section{Proof of Corollary 3}

We will need the following two results. The first identifies examples of doubled Farey graphs in the pants graph, and the second recalls a standard property of graph doubles. 
Lemma 7. Let $Q$ be a 2-handle multicurve on $\Sigma$. Then, the graph $\mathcal{P}_{Q}$ is isomorphic to the double of a Farey graph.

Proof. Let $Q_{1}$ and $Q_{2}$ be two disjoint rank 1 multicurves on $\Sigma$ such that $Q_{1} \cap Q_{2}$ is equal to $Q$. There exist natural retractions $\pi_{i}: \mathcal{P}_{Q} \rightarrow \mathcal{P}_{Q_{i}}$, for $i \in\{1,2\}$, and there exists an isomorphism $\phi: \mathcal{P}_{Q_{2}} \rightarrow \mathcal{P}_{Q_{1}}$. The map $f: \mathcal{P} \rightarrow \mathcal{P}_{Q_{1}} * \mathcal{P}_{Q_{1}}$ defined by $f(v)=\left(\pi_{1}(v), \phi \circ \pi_{2}(v)\right)$, for all vertices $v$ of $\mathcal{P}$, is thus an isomorphism. Finally, recall that $\mathcal{P}_{Q_{1}}$ is a Farey graph.

The following statement is standard, and we omit its proof.

Lemma 8. Let $G$ be a graph, and denote by $L$ a convex subgraph of $G$. Then, the graph double $L * L$ is a convex subgraph of $G * G$.

Proof of Corollary 3. Let $Q$ be any 2-handle multicurve on $\Sigma$, so that $\mathcal{P}_{Q}$ is isomorphic to the double of a Farey graph. Every bi-infinite geodesic $L$ is convex (but not necessarily totally geodesic), and the double $L * L$ is a convex subgraph of the totally geodesic subgraph $\mathcal{P}_{Q}$ of $\mathcal{P}$. Thus, $L * L$ is a convex subgraph of $\mathcal{P}$. Finally, $L * L$ is isomorphic to the Cayley graph of $\mathbb{Z} \oplus \mathbb{Z}$ with standard generating set, and as such is a plane.

For complexity 3 surfaces, the convex planes are all contained in 2-handle subgraphs.

Lemma 9. Suppose $\Sigma$ is of complexity 3, thus either the 6-holed sphere, the 3holed torus, or the closed surface of genus 2 . Then, every convex plane in $\mathcal{P}(\Sigma)$ is contained in a 2-handle subgraph.

Proof. A 4-cycle in a convex plane is not contained in a single Farey graph and as such uniquely determines a separating curve, namely that common to each of its four pants decompositions. Any adjacent 4-cycle intersects the original 4-cycle in an edge, and thus in at least one vertex. It follows that the same separating curve must be common to each of the second 4-cycle's four pants decompositions. The proof is completed by an induction, appealing to the connectivity of a plane.

\section{ACKNOWLEDGEMENTS}

The third author gratefully acknowledges the Department of Mathematical and Computing Sciences at the Tokyo Institute of Technology for its warm hospitality. The authors wish to thank Eiko Kin, Sadayoshi Kojima, Saul Schleimer, and Mitsuhiko Takasawa for several interesting conversations. The authors also wish to thank the referee for making numerous suggestions that greatly enhanced the exposition.

\section{REFERENCES}

1. J. Aramayona, H. Parlier, K. J. Shackleton, Totally geodesic subgraphs of the pants complex, Math. Res. Lett. 15, no. 2-3 (2008), 309-320. MR2385643 (2008m:57004)

2. J. A. Behrstock, C. Druţu, L. Mosher, Thick metric spaces, relative hyperbolicity, and quasiisometric rigidity, to appear in Math. Annalen.

3. J. F. Brock, The Weil-Petersson metric and volumes of 3-dimensional hyperbolic convex cores, J. Amer. Math. Soc. 16, no. 3 (2003), 495-535. MR.1969203 (2004c:32027)

4. J. F. Brock, B. Farb, Curvature and rank of Teichmüller space, Amer. J. Math., 128, no. 1 (2006), 1-22. MR2197066 (2006j:32013) 
5. J. F. Brock, H. A. Masur, Coarse and synthetic Weil-Petersson geometry: quasi-flats, geodesics, and relative hyperbolicity, Geom. Topol. 12 (2008), no. 4, 2453-2495. MR2443970

6. T. Chu, The Weil-Petersson metric in the moduli space, Chinese J. Math. 4 (1976), no. 2, 29-51. MR0590105 (58:28683)

7. A. E. Hatcher, W. P. Thurston, A presentation for the mapping class group of a closed orientable surface, Topology 19 (1980), 221-237. MR0579573 (81k:57008)

8. D. Margalit, Automorphisms of the pants complex, Duke Math. J. 121, no. 3 (2004), 457-479. MR 2040283 (2004m:57037)

9. H. A. Masur, Extension of the Weil-Petersson metric to the boundary of Teichmuller space, Duke Math. J. 43, no. 3 (1976), 623-635. MR0417456 (54:5506)

10. H. A. Masur, Y. N. Minsky, Geometry of the complex of curves II: Hierarchical structure, Geom. Funct. Anal. 10, no. 4 (2000), 902-974. MR1791145 (2001k:57020)

11. H. A. Masur, S. Schleimer, The pants complex has only one end, "Spaces of Kleinian groups" (eds. Y. N. Minsky, M. Sakuma, C. M. Series), Math. Soc. Lecture Note Ser. 329 (2006), 209-218. MR2258750 (2007g:57035)

12. S. A. Wolpert, Geodesic length functions and the Nielsen problem, J. Differential Geom. 25 (1987), no. 2, 275-296. MR880186 (88e:32032)

13. S. A. Wolpert, Noncompleteness of the Weil-Petersson metric for Teichmüller space, Pacific J. Math. 61 (1975), no. 2, 573-577. MR0422692(54:10678)

Department of Mathematics, National University of Ireland, Galway, Ireland

E-mail address: Javier.Aramayona@nuigalway.ie

$U R L$ : http://www.maths.nuigalway.ie

Institute of Geometry, Algebra and Topology, École Polytechnique Fédérale de Lausanne, BÂtiment BCH, CH-1015 Lausanne, Switzerland

E-mail address: hugo.parlier@a3.epfl.ch

$U R L:$ http://igat.epfl.ch

University of TOKyo IPMU, 5-1-5 Kashiwanoha, Kashiwa-Shi, Chiba 277-8568, Japan

E-mail address: kenneth.shackleton@ipmu.jp

E-mail address: kjs2006@alumni.soton.ac.uk

$U R L:$ http://member.ipmu.jp/kenneth.shackleton/ 